\title{
The Importance of Nanoparticles Addition in a Base Fluid Flow through a Porous Medium CIMPEAN Dalia Sabina
}

\author{
Department of Mathematics, Technical University of Cluj-Napoca, RO-400114 \\ $28^{\text {th }}$ Memorandumului Street, Cluj-Napoca, Romania \\ dalia.cimpean@math.utcluj.ro
}

Keywords: Nanoparticle, Porous medium, Mixed convection, Termophysical properties.

\begin{abstract}
The present study is focused on the mixed convection fluid flow through a porous medium, when a different amount of nanoparticles is added in the base fluid. The nanofluid saturates the porous matrix and different situations of the flow between two walls are presented and discussed. Alternatively mathematical models are presented and discussed. A solution of a system which contains the momentum, Darcy and energy equations, together with the boundary conditions involved, is given. The behavior of different nanofluids, such that $A u$-water, $A g$-water and Fe-water is graphically illustrated and compared with the previous results.

The research target is to observe the substantial increase of the thermophysical fluid properties, when the porous medium is saturated by a nanofluid instead of a classical Newtonian fluid.
\end{abstract}

\section{Introduction}

Because the conventional heat transfer fluids including oil, water and ethylene glycol mixture, are poor heat transfer fluids, an important and innovative technique, for improving heat transfer, was considered in the last years. The general technique, known as nanotechnology, is related in this study to nanofluids. The term nanofluid refers to regular fluids with suspending nanoscale particles. Choi [1] has introduced the first these notions. The nanofluids attracted the interest of many researchers, due to their potential for the high rate of heat exchange incurring either little or no penalty in the pressure drop. The nanoparticles are different from conventional particles (millimeter or micro-scale) in that they stay in suspension in the fluid and no sedimentation occurs.

Several studies on fluid flows through porous media were recently reported $([2,3,4])$, many other are related to nanofluids $([5,6,7])$. Some research paper include thermal conductivity (Kumar et al [8]), separated flow (Abu-Nada [9]) and convective heat transfer ( Tiwari and Das [10]). Eastman et al. [11] showed the particle size effect and the potential of nanofluids with smaller particles. Important references on nanofluids are given in the recent book by Das et al. [12] and in the review paper by Buongiorno [13].

The thermal performances of the nanofluids are required in many industrial fields, such as: electronic cooling applications, chemical sectors, ventilation, medical sectors for thermal therapy of cancer treatment, etc.

\section{Problem presentation}

Consider the mixed convection flow of a nanofluid in an inclined infinitely long two-dimensional channel bounded by parallel plane walls and filed with a fluid-saturated porous medium. The $x$ axis is considered up lengthways and the $y$ axis is oriented into the channel. The viscous fluid and the porous media properties are constant, except for the variation of density in the buoyancy term of the Darcy equation. The flow is fully developed and steady. An uniform upward streamwise velocity at the channel entrance is considered and an uniform heat flux $q_{w}$ is given at the walls, see Fig. 1. 

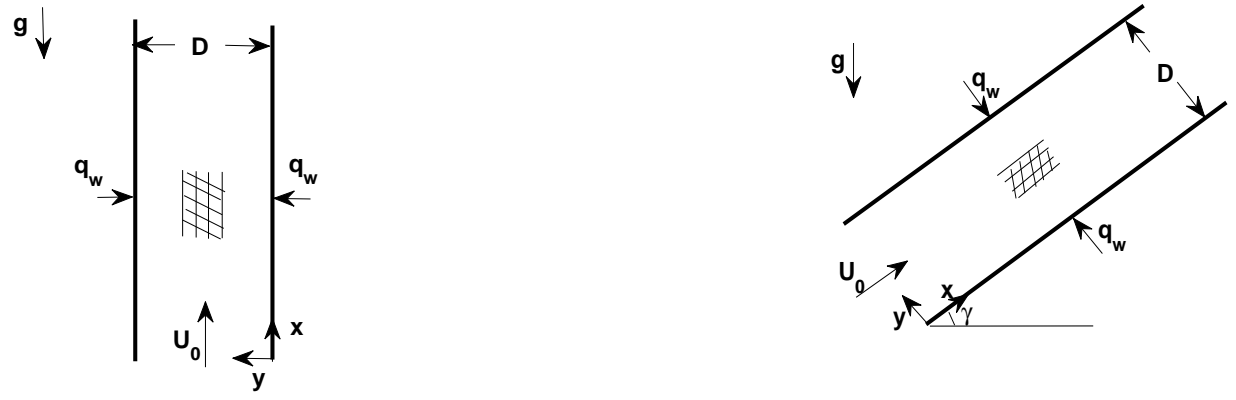

Fig. 1. A schematic diagram of the physical model for the vertical and inclined plates, respectively.

\section{Mathematical models}

The problem presented above is approached from two ways by taking into account important termophysical properties of the entire system and adapting, for the particular problem, mathematical models from the reported literature.

\section{Conservation equations for a porous medium saturated by a nanofluid}

We consider, first, a general model for a nanofluid instead the classical Newtonian fluid, see Cimpean [14]. It is assumed that nanoparticles are suspended in the nanofluid using either surfactant or surface charge technology. This prevents particles from agglomeration and deposition on the porous matrix. A two-dimensional problem of a steady flow is considered. We select a coordinate frame in which the $\mathrm{x}$-axis is aligned vertically upwards and take a vertical plate at $y=0$. At this boundary, the temperature $\mathrm{T}$ and the nanoparticle fraction $\phi$ take constant values $T_{w}$ and $\phi_{w}$, respectively. The ambient values, attained as y tends to infinity, are denoted by $T_{\infty}$ and $\phi_{\infty}$, respectively. The Oberbeck-Boussinesq approximation is employed. Homogeneity and local thermal equilibrium in the porous medium is assumed, as for the previous case. We consider a porous medium whose porosity is denoted by $\epsilon$ and permeability by $K$. The Darcy velocity is denoted by $\mathbf{v}=(u, v)$. The following four field equations, namely the conservation of total mass, momentum, thermal energy, and nanoparticles, respectively, are considered. The field variables are the Darcy velocity $\mathbf{v}$, the temperature $T$ and the nanoparticle volume fraction $\phi$ (see Nield and Kuznetsov [15]).

$$
\begin{aligned}
& \nabla \cdot \mathbf{v}=0 \\
& \frac{\mu}{K} \mathbf{v}=-\nabla p+\left\{\phi \rho_{p}+(1-\phi) \rho_{f}\left[1-\beta\left(T-T_{0}\right)\right]\right\} \mathbf{g} \\
& (\rho c)_{f} \mathbf{v} \cdot \nabla T=k_{m} \nabla^{2} T+\epsilon(\rho c)_{p}\left[D_{B} \nabla \phi \cdot \nabla T+\left(D_{T} / T_{\infty}\right) \nabla T \cdot \nabla T\right] \\
& \frac{1}{\epsilon} \mathbf{v} \cdot \nabla \phi=D_{B} \nabla^{2} \phi+\left(D_{T} / T_{\infty}\right) \nabla^{2} T
\end{aligned}
$$


Here $\rho_{f}, \mu$ and $\beta$ are the density, viscosity, and volumetric expansion coefficient of the fluid, while $\rho_{p}$ is the density of the particles. The gravitational acceleration is denoted by $\mathbf{g}$. We have introduced the effective heat capacity $(\rho c)_{m}$, and the effective thermal conductivity $k_{m}$ of the porous medium. The coefficients that appear in Eqs. (3) and (4) are the Brownian diffusion coefficient $D_{B}$ and the thermophoretic diffusion coefficient $D_{T}$. Details of the derivation of Eqs. (3) and (4) are reported by Buongiorno [13] and Nield and Kuznetsov [15].

Considering the velocity $\mathbf{v}=(u, v)$, the boundary conditions are:

$$
\begin{aligned}
& v=0, T=T_{w}, \phi=\phi_{w} \text { at } y=0 \\
& u=v=0, T \rightarrow T_{\infty}, \phi \rightarrow \phi_{w} \text { as } y \rightarrow \infty
\end{aligned}
$$

First model By following the considerations from the above paragraph, we consider now the fully developed mixed convection flow of a nanofluid between two parallel plates filled by a porous medium (see Fig.1) and write the governing equations of a nanofluid corresponding to the given problem, as:

$$
\begin{aligned}
& \frac{\partial u}{\partial x}+\frac{\partial v}{\partial y}=0 \\
& \frac{\partial p}{\partial x}+\frac{\mu}{K} u=-\left\{\phi \rho_{p}+(1-\phi) \rho_{f}\left[1-\beta\left(T-T_{0}\right)\right]\right\} g \sin \gamma \\
& \frac{\partial p}{\partial y}=-\left\{\phi \rho_{p}+(1-\phi) \rho_{f}\left[1-\beta\left(T-T_{0}\right)\right]\right\} g \cos \gamma \\
& u \frac{\partial T}{\partial x}=\alpha_{m} \frac{\partial^{2} T}{\partial y^{2}}+\epsilon \frac{(\rho c)_{p}}{(\rho c)_{f}}\left[D_{B} \nabla \phi \cdot \nabla T+\frac{D_{T}}{T_{0}}\left(\frac{\partial T}{\partial y}\right)^{2}\right] \\
& \left.\frac{1}{\epsilon} u \frac{\partial \phi}{\partial x}=D_{B}\left(\frac{\partial^{2} \phi}{\partial x^{2}}+\frac{\partial^{2} \phi}{\partial y^{2}}\right)+\frac{D_{T}}{T_{0}} \frac{\partial^{2} T}{\partial y^{2}}\right]
\end{aligned}
$$

The equations (7)-(11) are to be solved subject to the boundary conditions:

$$
v=0, \frac{\partial T}{\partial y}=-\frac{q}{k}, \phi=\phi_{1} \text { on } y=0 \quad \text { and } \quad v=0, \frac{\partial T}{\partial y}=\frac{q}{k}, \phi=\phi_{2} \text { on } y=D
$$

Second model The problem of the mixed convection flow of a nanofluid between parallel plane walls filled with a fluid-saturated porous medium is considered now from a different perspective (see the paper by Cimpean and Pop [16] ). We cosider the same conditions and the geometric configuration of the flow, see Fig.1. The nanofluid and porous media properties are constant except for the variation of density in the buoyancy term of the Darcy equation. The nanoparticle volume fraction, $\phi$, is considered constant for this model. Under these assumptions, with the use of the Darcy's law, the Boussinesq approximation and adopting the nanofluid model proposed by Tiwari and Das [10], the basic equations can be written in Cartesian coordinates $x$ and $y$ such that:

$$
\frac{\partial u}{\partial x}+\frac{\partial v}{\partial y}=0
$$




$$
\begin{aligned}
& \frac{\mu_{n f}}{K}\left(\frac{\partial u}{\partial y}-\frac{\partial v}{\partial x}\right)=\left[(1-\phi)(\rho \beta)_{f}+\phi(\rho \beta)_{p}\right] g\left(\frac{\partial T}{\partial y} \sin \gamma-\frac{\partial T}{\partial x} \cos \gamma\right) \\
& u \frac{\partial T}{\partial x}+v \frac{\partial T}{\partial y}=\alpha_{n f}\left(\frac{\partial^{2} T}{\partial x^{2}}+\frac{\partial^{2} T}{\partial y^{2}}\right)
\end{aligned}
$$

where $u$ and $v$ are the Cartesian velocity components, $T$ is the nanofluid temperature. The coefficients are $\beta$ the thermal expansion, $\rho$ the density, $K$ the specific permeability of the medium, $\mu_{n f}$ the viscosity of the nanofluid, $\phi$ the nanoparticle volume fraction and $\alpha_{n f}$ the thermal diffusivity of the nanofluid. The subscripts $f$ and $p$ correspond to the coefficients related to the fluid and nanoparticles, respectively. Also, the tilt angle, measured counterclockwise from the horizontal is denoted by $\gamma$ in the considered equations. The equations (13)-(15) are to be solved subject to the boundary conditions:

$$
\begin{gathered}
v=0, \frac{\partial T}{\partial y}=-\frac{q_{w}}{k_{n f}} \quad \text { on } \quad y=0 \\
v=0, \frac{\partial T}{\partial y}=\frac{q_{w}}{k_{n f}} \quad \text { on } \quad y=D
\end{gathered}
$$

where $q_{w}$ is the heat flux to the wall, $D$ is the channel width and $k_{n f}$ is the thermal conductivity of the nanofluid. Also, from the continuity equation (13), we have the forced flow condition,

$$
\int_{0}^{D} u d y=Q
$$

where $Q$ is a prescribed constant and represents the inflow at the channel entrance.

\section{Termophysical properties of the fluid and nanoparticles}

We consider throughout the paper, the initial conditions of the system at the ambient temperature, then the thermophysical properties of the base-fluid and nanoparticles are given in the Table 1 (see [17]).

Table 1

Thermophysical properties of fluid and metal nanoparticles at $20^{\circ}$ Celsius.

\begin{tabular}{|l|l|l|l|l|l|}
\hline $\begin{array}{l}\text { Physical } \\
\text { properties }\end{array}$ & $\begin{array}{l}\text { Fluid phase } \\
\text { (water) }\end{array}$ & Gold (Au) & $\begin{array}{l}\text { Silver } \\
(\mathrm{Ag})\end{array}$ & Iron $(\mathrm{Fe})$ & $\begin{array}{l}\text { Cooper } \\
(\mathrm{Cu})\end{array}$ \\
\hline$\rho\left(\mathrm{kg} / \mathrm{m}^{3}\right)$ & 998.2 & 19320 & 10524 & 7897 & 8954 \\
$k(\mathrm{~W} / \mathrm{mK})$ & 0.6 & 315 & 420 & 72.7 & 386 \\
$\beta(1 / \mathrm{K})$ & $2.07 \cdot 10^{-4}$ & $14 \cdot 10^{-6}$ & $18 \cdot 10^{-6}$ & $12 \cdot 10^{-6}$ & $17 \cdot 10^{-6}$ \\
$c_{p}(\mathrm{~J} / \mathrm{kgK})$ & 4183 & 129 & 234 & 452 & 383 \\
\hline
\end{tabular}

Also the density $\rho_{n f}$, the viscosity of the nanofluid $\mu_{n f}$ considering the viscosity of the base fluid $\mu_{f}$, the thermal conductivity $k_{n f}$ and thermal diffusivity $\alpha_{n f}$ of the nanofluid are given by the expressions

$$
\begin{gathered}
\rho_{n f}=\rho_{n f, 0}-\left[(1-\phi)(\rho \beta)_{f}+\phi(\rho \beta)_{p}\right]\left(T-T_{0}\right), \\
\alpha_{n f}=\frac{k_{n f}}{\left(\rho c_{p}\right)_{n f}}, \\
\left(\rho c_{p}\right)_{n f}=(1-\phi)\left(\rho c_{p}\right)_{f}+\phi\left(\rho c_{p}\right)_{p}, \\
\mu_{n f}=\frac{\mu_{f}}{(1-\phi)^{2.5}}, \\
k_{n f}=k_{f} \frac{\left(k_{p}+2 k_{f}\right)-2 \phi\left(k_{f}-k_{p}\right)}{\left(k_{p}+2 k_{f}\right)+\phi\left(k_{f}-k_{p}\right)}
\end{gathered}
$$




\section{An analytical solution}

Following the paper by Cimpean and Pop [16], we introduce the non-dimensional variables:

$$
X=\frac{x}{D}, \quad Y=\frac{y}{D}, \quad U=\frac{u}{U_{0}}, \quad \theta=\frac{\left(T-T_{0}\right)}{q D / k_{f}}
$$

where $U_{0}=Q / D$ is the velocity scale and $T_{0}$ is the uniform fluid temperature at the inflow. Then, we consider

$$
U=U(Y), \theta(X, Y)=C_{1} X+F(Y)
$$

and from the given equations (13)-(15) with the conditions (16) and (17), we have a third order ordinary differential equation:

$$
\frac{d^{3} F}{d Y^{3}}-(2 \lambda A \sin \gamma) \frac{d F}{d Y}+\frac{\alpha_{n f}}{\alpha_{f}} \frac{4 \lambda A \cos \gamma}{P e}=0
$$

which has to be solved, subject to the boundary conditions:

$$
\frac{d F}{d Y}=-1 \text { at } Y=0 ; \frac{d F}{d Y}=1 \text { at } Y=1
$$

In Eq.(21) we have denoted by $A$ the expression

$$
A=(1-\phi)^{2.5}\left[(1-\phi)+\phi \frac{(\rho \beta)_{p}}{(\rho \beta)_{f}}\right]
$$

Also, the mixed convection parameter $\lambda$ and the Péclet number $P e$, are given by

$$
\lambda=\frac{g(\rho \beta)_{f} K q D}{U_{0} \mu_{f} k_{f}}, P e=\frac{U_{0} D}{\alpha_{f}}
$$

Further, we will take into account the general case of the inclination of the channel $(\gamma>0)$ with the corresponding solutions (see Cimpean and Pop [16]). Then, the velocity profile is given by:

$$
U(Y)=\frac{\zeta}{2 \sinh \zeta}\left(1-\frac{2 \alpha_{n f} \cot \gamma}{\alpha_{f} P e}\right) \cosh \zeta Y+\frac{\zeta}{2 \sinh \zeta}\left(1+\frac{2 \alpha_{n f} \cot \gamma}{\alpha_{f} P e}\right) \cosh \zeta(1-Y)
$$

where $\zeta=\sqrt{2 \lambda A \sin \gamma}>0$.

The temperature component of the solution is obtained after applying the condition $\int_{0}^{1} F(Y) U(Y) d Y=0$ and has the form:

$$
\begin{aligned}
F(Y)= & \frac{1}{\zeta \sinh \zeta}\left(1-\frac{2 \alpha_{n f} \cot \gamma}{\alpha_{f} P e}\right) \cosh \zeta Y+\frac{2 \alpha_{n f} \cot \gamma}{\alpha_{f} P e} Y \\
& +\frac{1}{\zeta \sinh \zeta}\left(1+\frac{2 \alpha_{n f} \cot \gamma}{\alpha_{f} P e}\right) \cosh \zeta(1-Y)+C_{0}
\end{aligned}
$$

where the constant $C_{0}$ is given by

$$
\begin{aligned}
C_{0}= & -\frac{(\cosh \zeta+1)(\sinh \zeta+\zeta)}{2 \zeta \sinh ^{2} \zeta}-\frac{\alpha_{n f}}{\alpha_{f}} \frac{\cot \gamma}{P e}+\left(\frac{\alpha_{n f}}{\alpha_{f}}\right)^{2} \frac{2 \cot ^{2} \gamma}{P e^{2} \sinh ^{2} \zeta} \sinh ^{2} \zeta \\
& -\left(\frac{\alpha_{n f}}{\alpha_{f}}\right)^{2} \frac{2 \cot ^{2} \gamma}{P e^{2} \zeta \sinh ^{2} \zeta}(3 \sinh \zeta-\zeta)(\cosh \zeta-1)
\end{aligned}
$$




\section{Results and discussion}

It is important to discuss the case of the vertical channel $\left(\gamma=\frac{\pi}{2}\right)$. Here, the nanofluid behavior is not so different from the case of the clear Newtonian fluid (considered water for this paper).

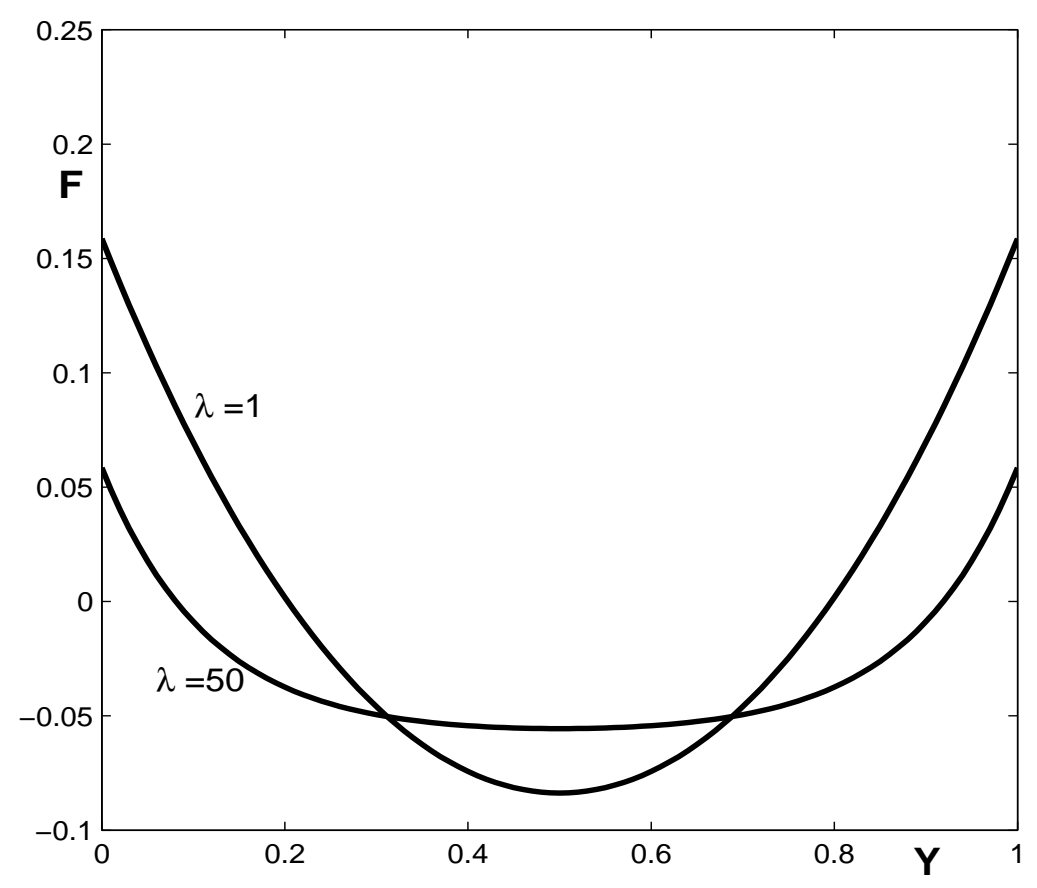

Fig. 2. Plots of $\mathrm{F}(\mathrm{Y})$ for $A g$ and $F e$ nanoparticles $(\phi=0.1)$ for $\gamma=\pi / 2, \lambda=1,50$ and $P e=1$.

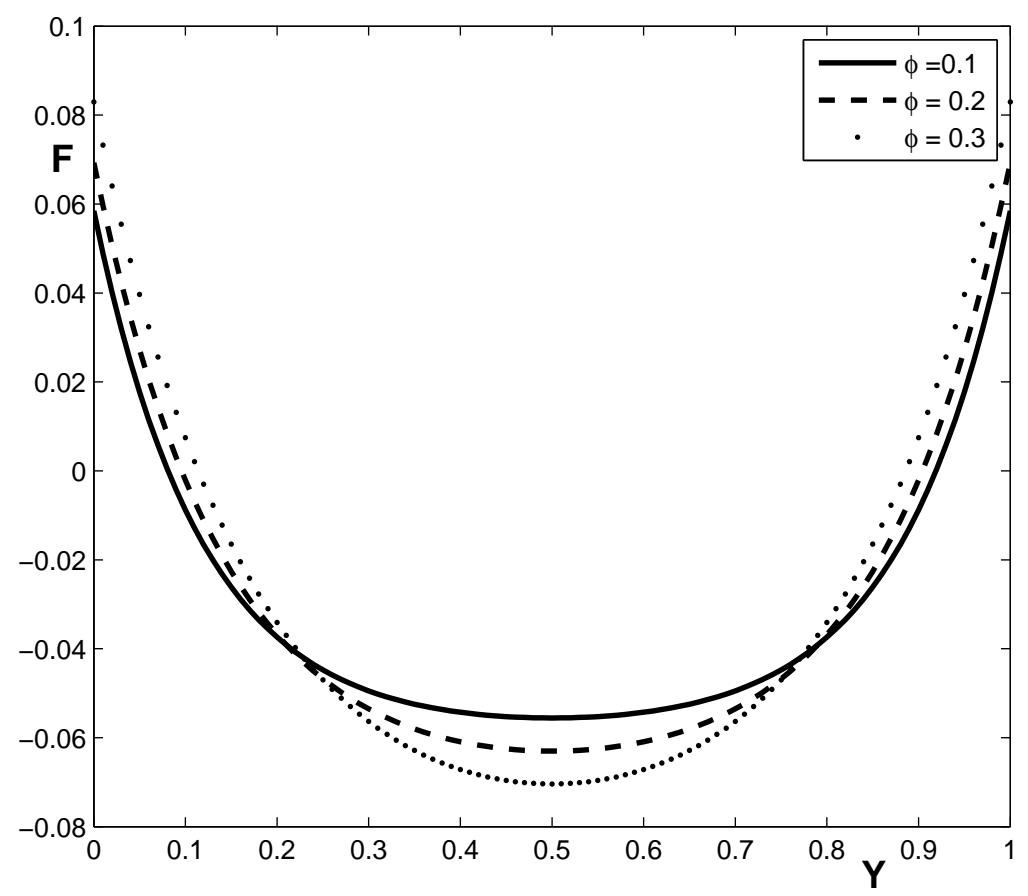

Fig. 3. Plots of $\mathrm{F}(\mathrm{Y})$ for different volume fraction of $F e$ nanoparticles $(\phi=0.1,0.2,0.3), \gamma=\pi / 2$, $\lambda=50$ and $P e=1$. 

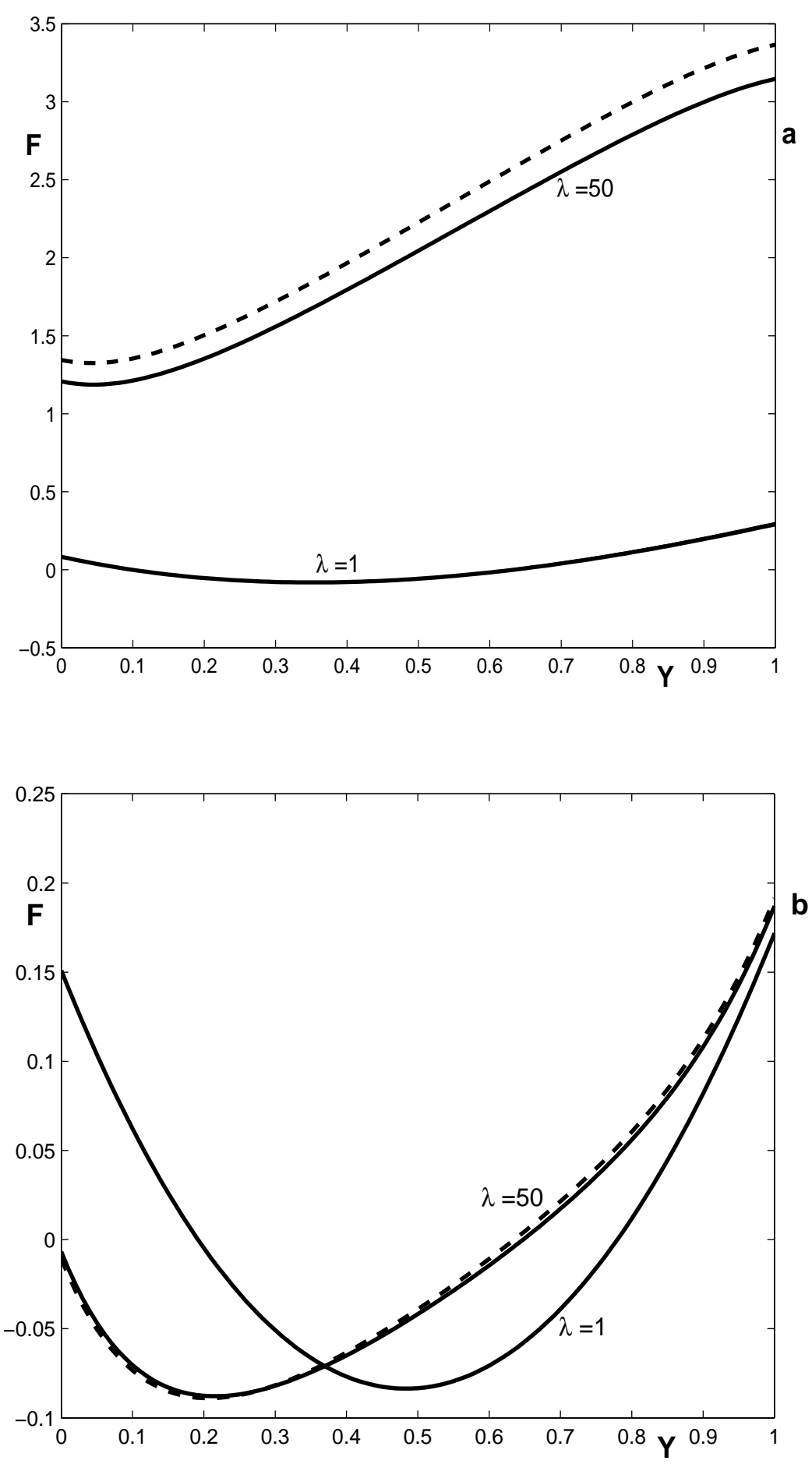

Fig. 4. Plots of $\mathrm{F}(\mathrm{Y})$ for $P e=1$ (a) and $P e=10$ (b), for $F e$ (line) and $A g$ (dots) nanoparticles $(\phi=0.1), \gamma=\pi / 4$ and $\lambda=1,50$.

In Fig. 2, the addition of different metal nanoparticles (iron and silver) does not change significantly the behavior of the temperature between the vertical walls. A very small change is observed when the nanoparticles volume fraction is increased, see Fig. 3.

The importance of the nanofluid is obvious when the plates are inclined. We consider for our results, the relevant inclination angle $\gamma=\frac{\pi}{4}$. 

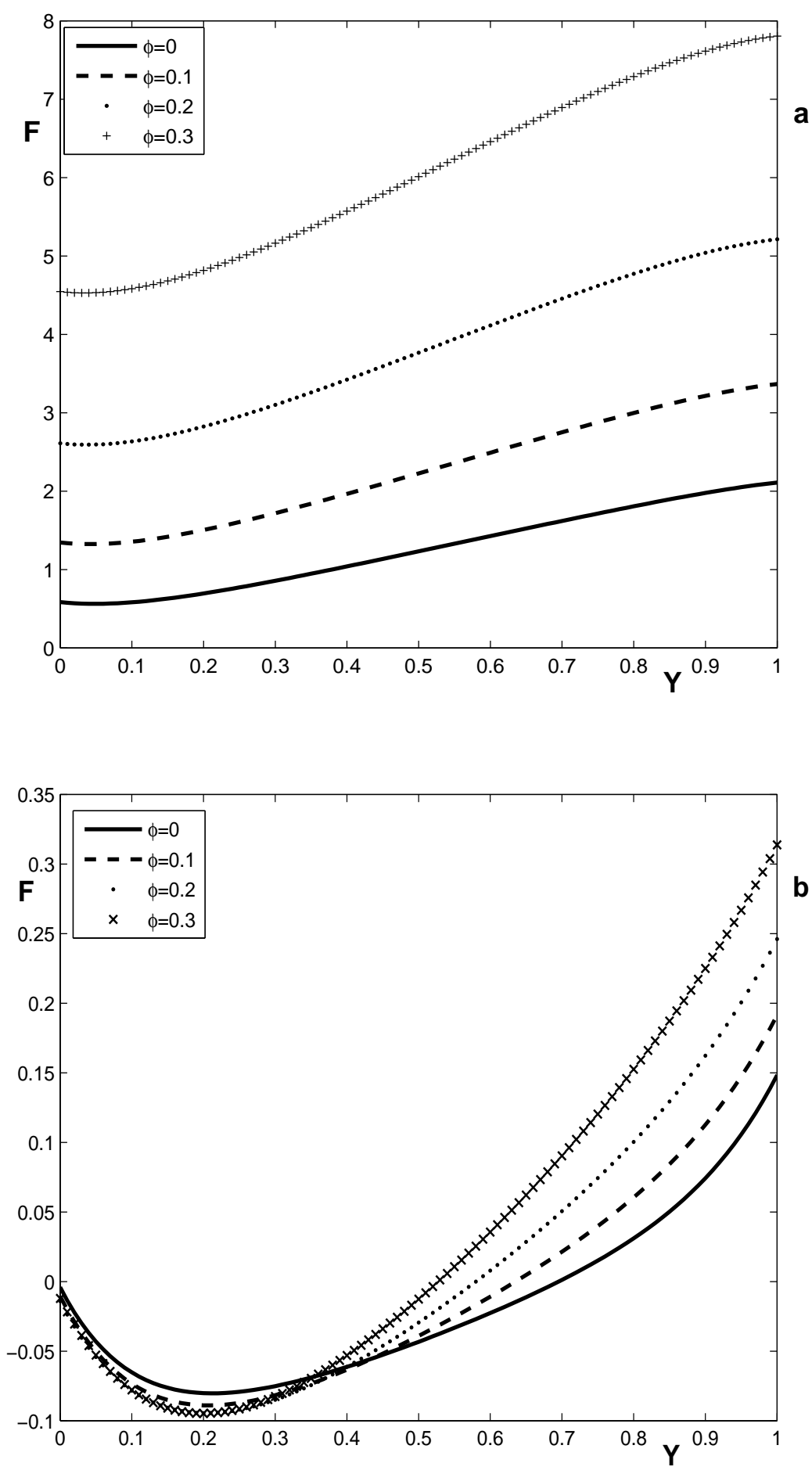

Fig. 5. The temperature profiles of $P e=1$ (a) and $P e=10$ (b) for different concentration of $\mathrm{Ag}$ nanoparticles $(\phi=0,0.1,0.2,0.3), \gamma=\pi / 4$ and $\lambda=50$.

It is worth to mention that, the small addition of nanoparticles $(\phi=0.1)$ in the water-based fluid, gives us an important increase of the thermal properties. Such that, in Fig. 4a, when the Péclet number is small, the temperature is increased when the mixed convection parameter increases, greater for Agnanoparticles. For high Péclet number (Fig. 4b), the nanofluid thermal properties are not improved comparing to the clear Newtonian fluid.

A very good performance is observed for the silver-nanofluid. In Fig. 5a is seen an important increase of the temperature when the nanoparticle volume fraction is increased. For high Péclet number 
( $P e \geq 10$ ), see Fig. 5b, this increase in temperature is relevant only between the middle and the upper wall of the channel. Also, for the case of clear Newtonian fluid $(\phi=0)$, is observed a very good agreement with the previous reported results, see Cimpean et al. [18].

In Fig. 6, a comparison of different nanofluids is seen. The best performance is observed for goldnanoparticles addition in the water-base fluid, when the thermophysical properties of the fluid are greately increased. The second performance is for silver and is followed by cooper and iron nanoparticles concentration.

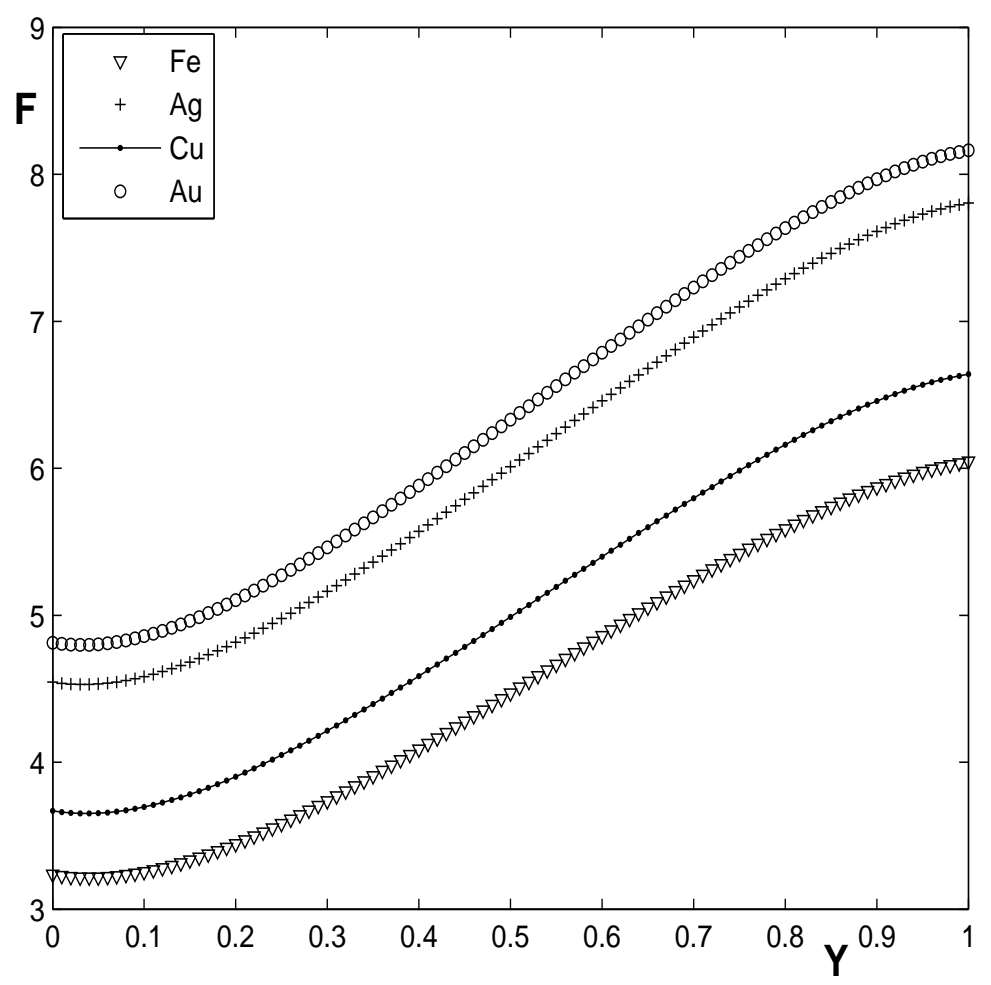

Fig. 6. Plots of F(Y) for different nanofluids (Fe-water, Ag-water, Cu-water, Au-water) for $\phi=0.3, \gamma=\pi / 4, \lambda=50$ and $P e=1$.

\section{Conclusions}

The present paper investigates the performances of different types of nanofluids through a porous medium, between two parallel plates. A steady mixed convection flow is considered and initial velocity and temperature are given. Two mathematical models are presented and discussed. An analytical solution is used to observe the behavior of the temperature of the flow for different given conditions. The importance of improving the clear Newtonian fluid with metal nanoparticles is discussed. The thermal performances of iron-water, silver-water, cooper-water and gold-water nanofluids are presented in figures and compared. The best performance is obtained for gold nanoparticles addition in the water-based fluid.

\section{Acknowledgements}

This paper was supported by the project "Development and support of multidisciplinary postdoctoral programmes in major technical areas of national strategy of Research - Development - Innovation" 4DPOSTDOC, contract no. POSDRU/89/1.5/S/52603, project co-funded by the European Social Fund through Sectoral Operational Programme Human Resources Development 2007-2013. 


\section{References}

[1] S.U.S. Choi, Enhancing thermal conductivity of fluids with nanoparticles. In: Siginer, D.A.,Wang, H.P. (eds.) Development and Applications of Non-Newtonian Flows, vols. MD-231, FED-66, pp. 99-105. ASME, New York (1995).

[2] I. Pop, D.B. Ingham, Convective Heat Transfer: Mathematical and Computational Modeling of Viscous Fluids and Porous Media, Pergamon, Oxford, 2001.

[3] A. Bejan, I. Dincer, S. Lorente, A.F. Miguel, A.H. Reis: Porous and Complex Flow Structures in Modern Technologies Springer, New York, 2004.

[4] P. Vadasz, Emerging Topics in Heat and Mass Transfer in Porous Media, Springer, 2008.

[5] Abu-Nada, E., Oztop, H.F.: Effects of inclination angle on natural convection in enclosures filled with $\mathrm{Cu}$-water nanofluid. Int. J. Heat Fluid Flow 30, 669-678 (2009).

[6] S. Ahmad, I. Pop: Mixed convection boundary layer flow from a vertical flat plate embedded in a porous medium filled with nanofluids: Int. Commun. Heat Mass Transf. doi:10.1016/j.icheatmasstransfer.2010. 06.004 (2010).

[7] V. Trisaksri, S. Wongwises: Critical review of heat transfer characteristics of nanofluids, Renew. Sustain. Energy Rev. 11, 512-523 (2007).[-23pt]

[8] S. Kumar, S.K. Prasad, J. Banerjee: Analysis of flow and thermal field in nanofluid using a single phase thermal dispersion model. Appl. Math. Model. 34, 573-592 (2010).

[9] E. Abu-Nada: Application of nanofluids for heat transfer enhancement of separated flows encountered in a backward facing step. Int. J. Heat Fluid Flow 29, 242-249 (2008).

[10] R.K. Tiwari, M.K. Das: Heat transfer augmentation in a two-sided lid-driven differentially heated square cavity utilizing nanofluids, Int. J. Heat Mass Transf. 50, 2002-2018 (2007).

[11] J.A. Eastman, S.U.S. Choi, S. Li, W. Yu, L. Thompson: Anomalously increased effective thermal conductivity of ethylene glycol-based nanofluids containing copper nanoparticles, J. Appl. Phys. Lett. 78, 718-720 (2010).

[12] S.K. Das, S.U.S. Choi, W. Yu, T.Pradet: Nanofluids: Science and Technology Wiley, New Jersey (2007).

[13] J. Buongiorno: Convective transport in nanofluids. ASME J. Heat Transf. 128, 240-250 (2006).

[14] D.S. Cimpean, An Analysis Of A Fluid Flow In A Channel With Uniform Heated Walls, Acta Technica Napocensis, Series: Applied Mathematics And Mechanics, Vol 2, No 54, 293--298 (2011).

[15] D.A. Nield, A.V. Kuznetsov: Thermal instability in a porous medium layer saturated by a nanofluid: Int. J. Heat Mass Transfer 52, 5796-5801 (2009).

[16] D.S. Cimpean, I. Pop: Fully developed mixed convection flow of a nanofluid through an inclined channel filled with a porous medium, Int. J. Heat Mass Transfer, vol. 55, Issue 4, pp. 907--914 (2012).

[17] Glenn Elert, The Physics Factbook, An educational website, 2000.

[18] D. Cimpean, I. Pop, D. Ingham, J. Merkin, Fully developed opposing mixed convection flow between inclined parallel plates field with a porous medium: Transport in Porous Media Int J, 77, 87--102 (2009). 